\title{
Changes in Postural Control in Mastectomized Women
}

\author{
Thais Montezuma, Elaine Caldeira de Oliveira Guirro*, \\ Maíta Mara de Oliveira Lima Leite Vaz, Sebastián Vernal \\ Department of Biomechanics, Medicine and Rehabilitation of the Locomotor Apparatus, \\ Faculty of Medicine of Ribeirão Preto, University of São Paulo, São Paulo, Brazil \\ Email: ecguirro@fmrp.usp.br
}

Received 16 March 2014; revised 15 April 2014; accepted 22 April 2014

Copyright (C) 2014 by authors and Scientific Research Publishing Inc.

This work is licensed under the Creative Commons Attribution International License (CC BY). http://creativecommons.org/licenses/by/4.0/

(c) (i) Open Access

\begin{abstract}
Background: Women undergoing mastectomy may have postural asymmetries due to mutilation, culminating in changes in postural balance. Aim: This study aimed to evaluate the influence of the mastectomy in the postural control of women undergoing surgical treatment of breast cancer. Methods: We evaluated 40 volunteers divided into two groups: women undergoing mastectomy (MG) with a mean age of 51.45 (SD 6.49) years old and mean BMI of 30.71 (SD 5.21 ) $\mathrm{kg} / \mathrm{m}^{2}$, and a control group of women without the disease (CG) with a mean age of 50.50 (SD 7.85) years old and mean BMI of 30.50 (SD 5.77). Static balance was assessed using the displacement of the center of pressure (COP), with voluntary bipedal support with eyes open and closed on a force platform. Statistical analysis was performed using the software SPSS 21.0 with a significance level of $5 \%$. Results: The MG showed an increase in the displacement area along the $x$-axis $(P=0.003)$ and total displacement $(P<0.001)$ with eyes open $(E 0)$, and an increase in the $x$-axis $(P=0.002)$ and total displacement $(P<0.001)$ with eyes closed $(E C)$. Velocity in the $x$-axis $(P<0.001), y$-axis $(P<0.001)$ and total velocity $(P<\mathbf{0 . 0 0 1})$ was higher with EO than EC. A rise in velocity was also observed in the $x$ - and $y$-axis, and in total velocity with EC, when compared with the CG $(P<0.001)$. Displacement was higher for all variables in both groups with EC $(P<0.001)$. Conclusions: Surgery for unilateral mastectomy may significantly alter postural control in women with breast cancer.
\end{abstract}

\section{Keywords}

Balance, Breast Neoplasms, Mastectomy, Physical Therapy Assistants

\section{Introduction}

Breast cancer is the most common type of cancer in Europe (464,000 cases) [1]. In 2013 its mortality rate was

\footnotetext{
*Corresponding author.
} 
the highest among women with cancer, with an index of 15\%, demonstrating a 7\% drop from 2009 in the European Union [2]. It is also considered the leading cause of death due to cancer in women among developing countries [3]. Nevertheless, breast cancer mortality has shown a significant decrease in Slovenia, Croatia and Malta [4].

Mastectomy is one of the most commonly recommended treatments for breast cancer, despite the fact that it may carry several comorbidities, including postural changes, such as a greater angle of trunk inclination, higher positioning of the scapula on the affected side in the frontal plane, greater angle of pelvis twisting, steepening the trunk forward, more abducted scapula and changes in the thoracic kyphosis and lordosis lumbar angles [5] [6].

A trunk static imbalance can occur, negatively affecting good posture. Proper and regular use of external breast prosthesis (EBP) can minimize postural changes and make the rehabilitation process more effective [5], given that static posture can be significantly influenced by the size of breasts [6].

Musculoskeletal mechanisms have been developed to compensate for changes to the physical characteristics of the human body. Changes to a body segment or posture result in displacement of the center of gravity, which can lead to instability [7].

Sustaining the hypothesis that surgery for unilateral mastectomy may influence postural control, the aim of the study is to assess the influence of mastectomy on static equilibrium in women with breast cancer.

\section{Methods}

\subsection{Patients}

A total of 60 women were elected for inclusion (Figure 1). Forty were enrolled in the study and divided into two homogeneous groups: a women with mastectomy group (MG) composed of women with unilateral mastectomy (Madden type), a mean age of 51.45 (SD 6.49) years old and mean BMI of 30.71 (SD 5.21) kg/ $\mathrm{m}^{2}$; and a control group with women without cancer with a mean age of 50.50 (SD 7.85) years old and mean BMI of 30.50 (SD 5.77) $\mathrm{Kg} / \mathrm{m}^{2}$. Women with mastectomies were treated at the Teaching, Research and Rehabilitation Assistance Center for Mastectomized Women-University of São Paulo, Brazil. The study was approved by the Research Ethics Committee of the institution (Protocol 10734/2012) and all women signed an informed consent form before enrollment.

Women were excluded who were previously diagnosed clinically (proven by medical specialist) with severe

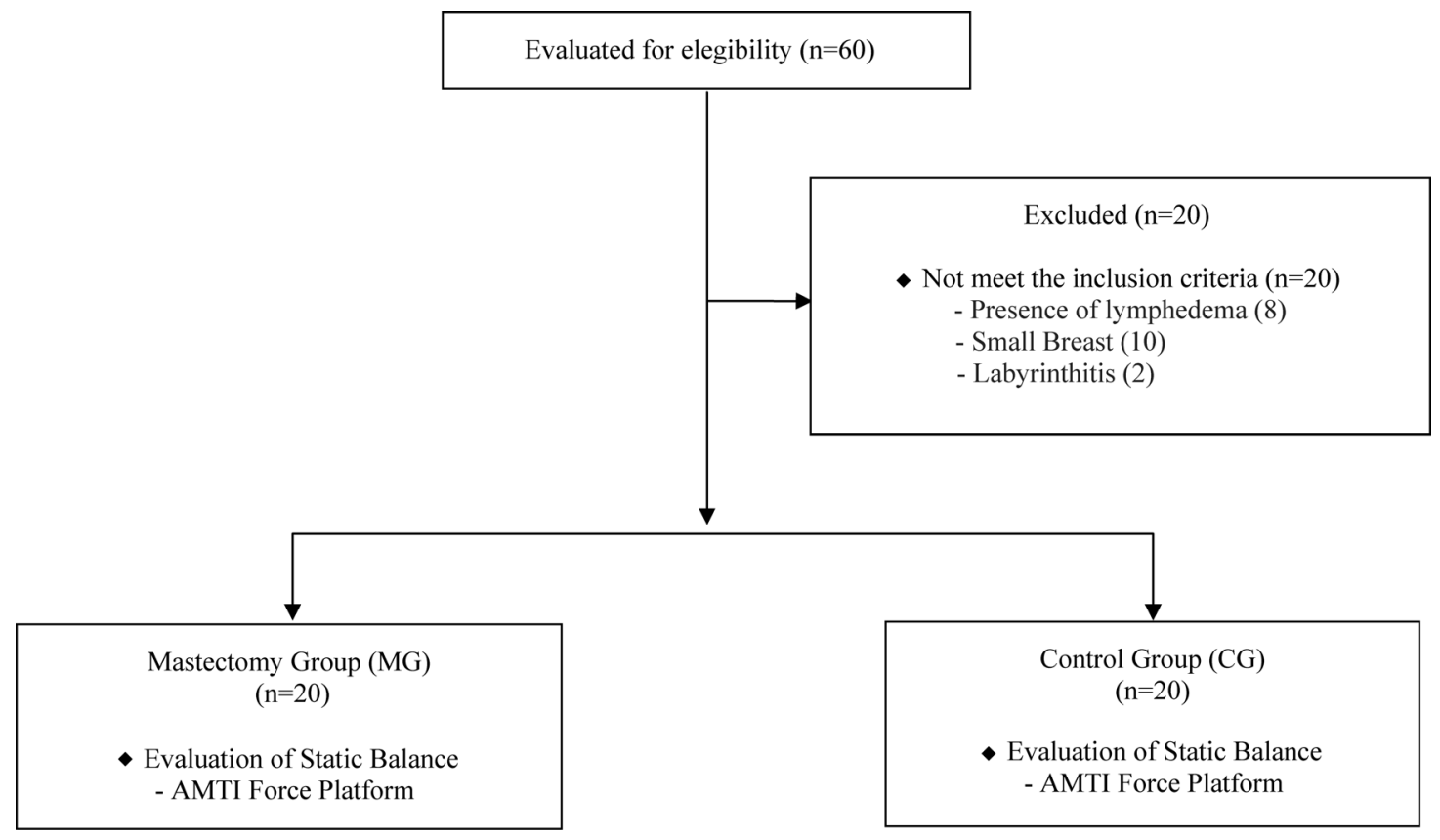

Figure 1. Consort flow chart distribution groups. 
degenerative diseases (arthrosis, arthritis, etc.) or any postural or equilibrium amendment were unable to remain standing erect, had bilateral mastectomy or lymphedema (proven by volumetric measure), were in chemo or radiotherapy, were using an internal mammary prosthesis or an expander in the breast region, or had small breasts (proven by Sacchini index) [8].

\subsection{Measurement}

Both groups underwent standard prior medical record review, examination of upper limb volume using the formula of the truncated cone [9], and review of breast size through the Sacchini index, defining three categories: less than $9 \mathrm{~cm}$-small breast, 9 to $11 \mathrm{~cm}$ —medium breast, and greater than $11 \mathrm{~cm} —$ big breast [8].

Ground force reaction was capture by Force Platform OR 06.07.1000 (Advanced Mechanical Technologies, Inc-AMTI, Watertown, USA) with an ICC $=96$ of four sensors [10], with dimensions $464 \times 508 \times 82.5 \mathrm{~mm}$. The BioDynamicsBr and BioDynamicsBr Analysis software were used for data collection and analysis of data, respectively. The main variables analyzed were the maximum displacement in the $\mathrm{x}$-axis (MDx), maximum displacement in the y-axis (MDy), total displacement (Dt), the mean velocity in the $\mathrm{x}$-axis (MVx), mean velocity in the $\mathrm{y}$-axis (MVy), and mean total velocity (MVt) of center of pressure (COP). Assessment of static balance on the force platform was assessed in the frequency of $100 \mathrm{~Hz}$, with three replications maintained for 60 seconds with eyes open (EO) and eyes closed (EC), bipedal support, in random sequence defined by simple drawing. For the EO evaluation, the participant should looked fixedly toward a dot placed one meter away, adjustable to eyes' height [11]-[13].

\subsection{Statistical Analysis}

The sample size was estimated at 16 volunteers for each group intervention based on Rostkowska, Bak and Samborski's study (2006), calculated by Graphpad Statemate software, with power of $80 \%$ and an alpha error of 0.05 . Data were summarized using frequency tables, summary statistics, confidence intervals, and $\mathrm{p}$ values, as appropriate. Demographic data are expressed as means \pm SD for continuous variables and frequencies and percentages for categorical variables. The preferred method of analysis for continuous variables was parametric, nonparametric analysis methods were used only if parametric assumptions could not be satisfied. Parametric model assumptions were assessed using the normal—plot or Shapiro-Wilk test to verify normality and Levene's test to verify homogeneity of variances. Continuous variables were compared between the two groups using a two sample (unpaired) $t$ test and between two measures of the same volunteer using at paired $t$ test. For continuous variables with skewed distribution the nonparametric Mann-Whitney $U$ test was used to compare two groups. Categorical variables between the groups were contrasted using the Chi-square $\left(\mathrm{x}^{2}\right)$ test, although Fisher's exact test was used when data were sparse. Significance was set at $P<0.05$, using two-tails comparison. All reported $P$-values are rounded to three decimal places. The statistical analysis was performed using the Statistical Package for Social Sciences (SPSS version 21.0, IBM ${ }^{\circledR}$, USA).

\section{Results}

Regarding the general characteristics of the volunteers there was no significant difference between the groups, demonstrating the homogeneity of the sample (Table 1).

Results of the static equilibrium assessment on the force platform with eyes open showed an increase in the MG for maximum displacement in the $\mathrm{x}$-axis $(P=0.003)$ and total displacement $(P<0.001)$ of center of pressure when compared to CG. With eyes closed was also found an increase in the MG for maximum displacement in the $\mathrm{x}$-axis $(P=0.002)$ and total displacement $(P<0.001)$ of center of pressure compared to CG (upper Table 2). Comparing each item for eyes open versus eyes closed in both groups, there were significant differences between the two for maximum displacement in the x-axis, maximum displacement in the y-axis and total displacement of center of pressure, with a higher displacement with eyes closed (lower Table 2).

Assessment results of mean velocity on the force platform demonstrated a velocity augmentation, even with eyes open or eyes closed, for mean velocity in the $x$-axis, mean velocity in the $y$-axis and mean total velocity of center of pressure in GM when compared to CG $(P<0.001)$, upper Table 3. In the analysis eyes open against eyes closed for each group, there was a significant difference for mean velocity in the $x$-axis, mean velocity in the $y$-axis and mean total velocity of center of pressure, with a higher velocity with eyes closed (lower Table 3). 
Table 1. Characteristics of volunteers.

\begin{tabular}{ccccc}
\hline & MG $(N=20)$ & CG $(N=20)$ & Total \\
\cline { 2 - 4 } & Mean $(S D)$ & Mean $(S D)$ & $P^{1}$ & Mean $(S D)$ \\
\hline Age (years) & $51.45(6.49)$ & $50.50(7.85)$ & 0.67 & $50.98(7.13)$ \\
BMI (Kg/m $\left.{ }^{2}\right)$ & $30.71(5.21)$ & $30.50(5.77)$ & 0.90 & $30.61(5.43)$ \\
Surgery time (months) & $5.80(5.76)$ & - & - & $5.80(5.76)$ \\
Index Sacchini (cm) & $12.18(2.07)$ & $12.45(2.94)$ & & $12.31(2.52)$ \\
Big breast & $N(\%)$ & $N(\%)$ & $0.73(\%)$ & $15(37.5)$ \\
Medium breast & $6(30)$ & $9(45)$ & & $25(62.5)$ \\
\hline
\end{tabular}

Abbreviations: MG, mastectomy group; CG, control group; SD, standard deviation; BMI, body mass index. ${ }^{1} \mathrm{MG}$ versus CG.

Table 2. Maximum displacement in the $\mathrm{x} / \mathrm{y}$-axis and total displacement (meters) of center of pressure in the force platform.

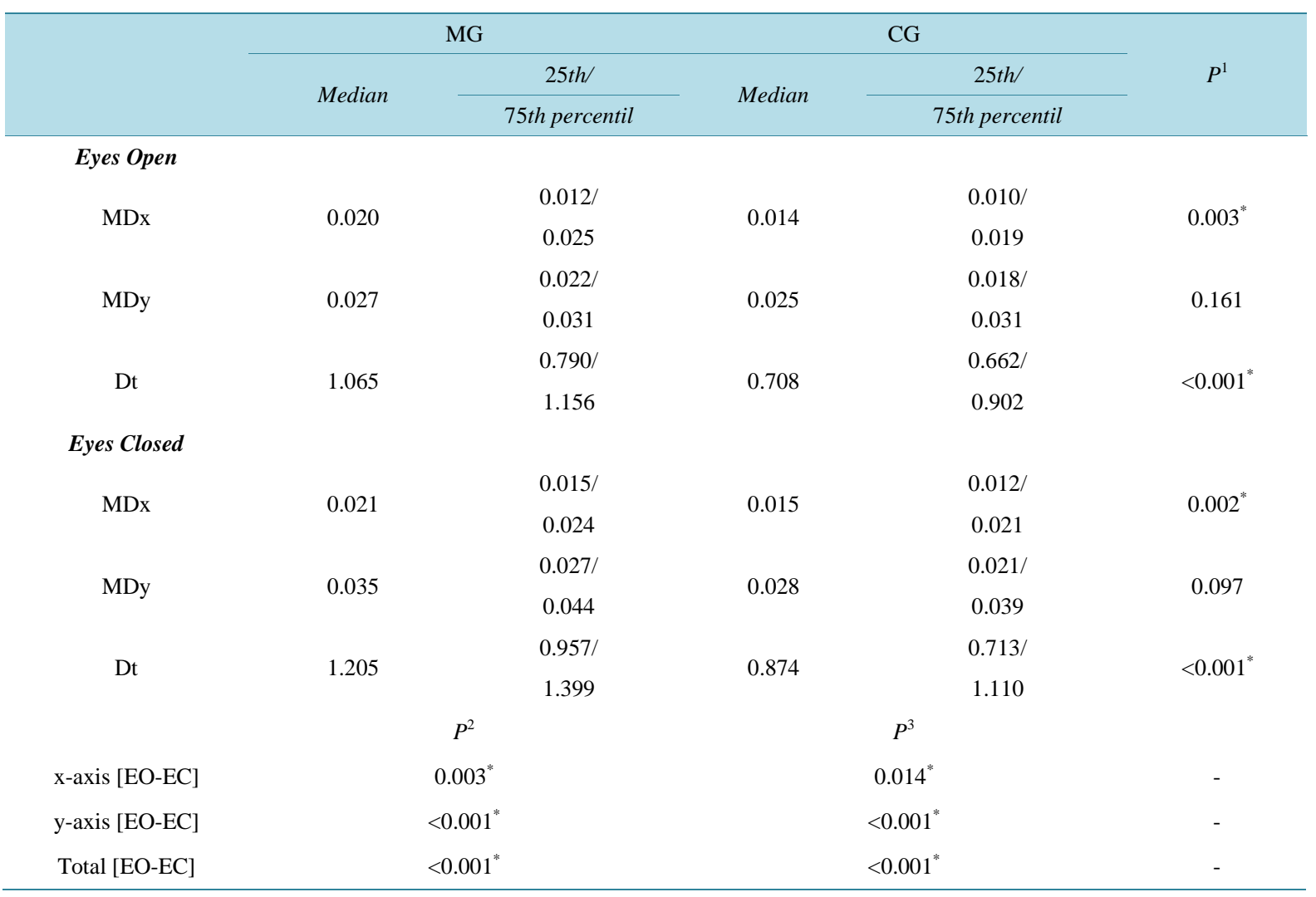

Abbreviations: MG, mastectomy group; CG, control group; MDx, maximum displacement in forward-backward direction; MDy, maximum displacement in lateral direction; Dt, total displacement; EO, eyes open; EC, eyes closed. ${ }^{1} \mathrm{MG}$ versus CG; ${ }^{2}$ Eyes open versus Eyes closed of MG; ${ }^{3}$ Eyes Open versus Eyes closed of CG. ${ }^{*} P<0.05$.

\section{Discussion}

As hypothesized, the surgery for unilateral mastectomy may influence postural control. Women undergoing mastectomy showed an increase in displacement and velocity of the center of pressure on the force platform when compared to women in the control group.

Breast hypertrophy may affect some muscles including pectoralis major, pectoralis minor, rhomboids and trapezius (middle and lower portion), leading to a muscle weakness proven by force test [14]. Muscle modifications can also be found in women who underwent mastectomy with strength reduction in the upper trapezius and 
Table 3. Mean velocity in the $\mathrm{x} / \mathrm{y}$-axis and total displacement (meters) of center of pressure in the force platform.

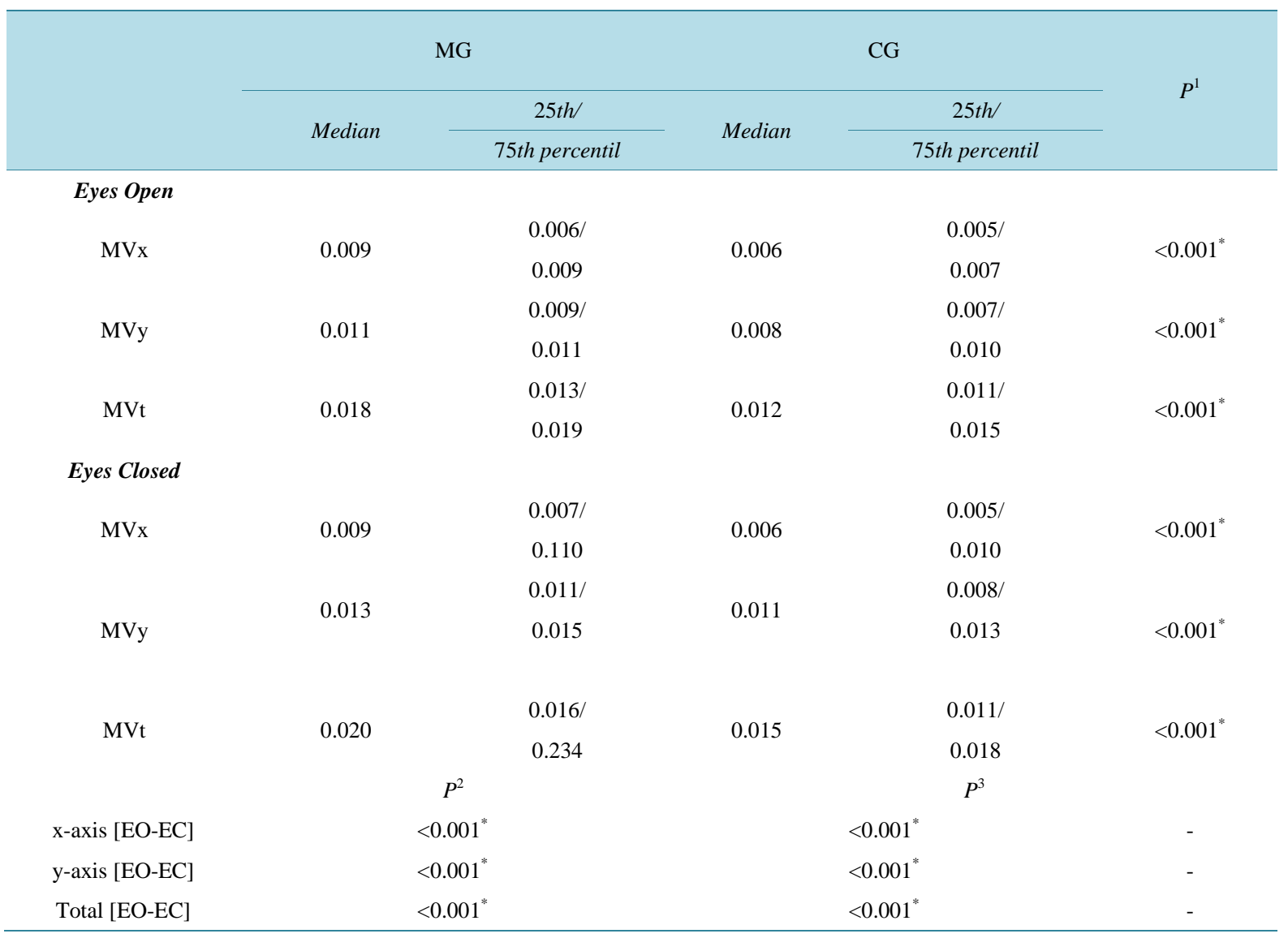

Abbreviations: MG, mastectomy group; CG, control group; MVx, mean velocity in forward-backward direction; MVy, mean velocity in lateral direction; MVt, mean total velocity; EO, open eyes; EC, eyes closed. ${ }^{1} \mathrm{MG}$ versus CG; ${ }^{2}$ Eyes open versus Eyes closed of MG; ${ }^{3}$ Eyes Open versus Eyes closed of CG. ${ }^{*} P<0.05$.

rhomboids [15].

Ciesla and Polom [16] evaluated the posture of women pre and post mastectomy surgery with a follow up at the $6^{\text {th }}, 12^{\text {th }}, 18^{\text {th }}$ and $24^{\text {th }}$ month after unilateral mastectomy; the results showed a significant change of posture in all follow up months, especially at the $18^{\text {th }}$ and $24^{\text {th }}$ months. Other authors have observed changes in posture and spine function in women who underwent mastectomy [5].

There is evidence that mastectomy can lead to postural alterations, causing variations in the center of gravity and, consequently, changes in static equilibrium, as observed in this study. The biomechanical parameters of reaction force and plantar distribution are modified in both dynamic and static forms [17].

One study evaluating factors related to falls in postmenopausal women with breast cancer surgery suggests that balance disorders of vestibular origin and delays in detecting low-contrast visual stimuli are associated with falls in these women. A relationship was also observed between falls and chemotherapy treatment, an association not observed with the use of adjuvant endocrine therapy [18]. Chemotherapy is a negative factor affecting postural control in women with breast cancer, increasing instability and pressure center score [19]. In this study, women were not included in the period of chemotherapy, but it is possible to observe that the mastectomy surgery is also an important factor increasing instability and changes in the pressure center.

Posture analysis in women after reductive mammoplasty, showed a significant decrease in the anterior tilt angle of the pelvis, and forward tilt flexion of the spine [20]. Thus, could be inferred that breast size influences the postural biomechanics. When assessing static balance in women with breast reduction, there is a decline in both, area and velocity displacement [21]. Barbosa et al. [22] showed an increase in the area and velocity displacement of the pressure center of women with breast hypertrophy. The fact that changes to breast sizes affect balance can justify the postural control variations in post-mastectomy women found in this study. 
Analyzing the pressure center on pilots with closed eyes, there was a 32\% change in the displacement area and a 50\% change in velocity, showing that visual occlusion is a negative factor for static equilibrium [23]. This is in agreement with the results found in both groups in our study.

The increased weight distribution causes a unilateral postural asymmetry and displacement of the mass center, directly affecting the larger unilateral weight, and providing poorer postural stability and augmentation of the displacement velocity of the pressure center [24]. A greater difference in the distribution of body mass would lead to a greater rate of medial-lateral displacement, increasing the risk of falls. Since mastectomy surgery causes a larger number of postural asymmetries and mass center displacements, it could be deduced that more significant contralateral breast surgery would lead to worse postural control.

Genthon and Rougier [25] evaluated pressure center behavior in the body weight distribution with different loads and noted that the mechanism of control decreases with increasing postural asymmetry, affecting a change in pressure center displacement toward medial-lateral, agreeing with the results obtained in the present study.

These findings are an innovation with respect to the balance in women with mastectomies, describing that mastectomy surgery may result in a loss of postural stability in women with breast cancer and highlighting the importance of rehabilitation for these women. It would be interesting to evaluate the effects of balance exercises in women undergoing surgical treatment for breast cancer.

\section{Conclusion}

Undergoing surgery for unilateral mastectomy results in postural control changes in women with breast cancer.

\section{Conflict of Interest}

The authors do not have any conflict of interest to disclose.

\section{Acknowledgements}

Foundation for Research Support of the State of São Paulo (FAPESP).

\section{References}

[1] Ferlay, J., Steliarova-Foucher, E., Lortet-Tieulent, J., Rosso, S., Coebergh, J.W., Comber, H., Forman, D. and Bray, F. (2013) Cancer Incidence and Mortality Patterns in Europe: Estimates for 40 Countries in 2012. European Journal of Cancer, 49, 1374-1403. http://dx.doi.org/10.1016/j.ejca.2012.12.027

[2] Malvezzi, M., Bertuccio, P., Levi, F., La Vecchia, C. and Negri, E. (2013) European Cancer Mortality Predictions for the Year 2013. Annals of Oncology, 24, 792-800. http://dx.doi.org/10.1093/annonc/mdt010

[3] Jemal, A., Bray, F., Center, M.M., Ferlay, J., Ward, E. and Forman, D. (2011) Global Cancer Statistics. CA: A Cancer Journal for Clinicians, 61, 69-90. http://dx.doi.org/10.3322/caac.20107

[4] Znaor, A., Van der Hurk, C., Primic-Zakelj, M., Agius, D., Coza, D., Demetriou, A., Dimitrova, N., Eser, S., Karakilinc, H., Zivkovic, S., Bray, F. and Coebergh, J.W. (2013) Cancer Incidence and Mortality Patterns in South Eastern Europe in the last Decade: Gaps Persist Compared with the Rest of Europe. European Journal of Cancer, 49, 16831691. http://dx.doi.org/10.1016/j.ejca.2012.11.030

[5] Rostkowska, E., Bak, M. and Samborski, W. (2006) Body Posture in Women after Mastectomy and Its Changes as a Result of Rehabilitation. Advances in Medical Sciences, 51, 287-297.

[6] Findikcioglu, K., Findikcioglu, F., Ozmen, S. and Guclu, T. (2007) The Impact of Breast Size on the Vertebral Column: A Radiologic Study. Aesthetic Plastic Surgery, 31, 23-27. http://dx.doi.org/10.1007/s00266-006-0178-5

[7] Danis, S.C.G., Krebs, D.E., Mgill-body, K. and Sarmann, S. (1998) Relationship between Standing Posture and Stability. Physical Therapy, 78, 502-517.

[8] Sacchini, V., Luini, A., Tana, S., Lozza, L., Galimberti, V., Merson, M., Agresti, R., Veronesi, P. and Greco, M. (1991) Quantitative and Qualitative Cosmetic Evaluation after Conservative Treatment for Breast Cancer. European Journal of Cancer, 27, 1395-1400. http://dx.doi.org/10.1016/0277-5379(91)90019-A

[9] Sander, A.P., Hajer, N.M., Hemenway, K. and Miller, A. (2002) Upper-Extremity Volume Measurements in Women with Lymphedema: A Comparison of Measurements Obtained via Water Displacement with Geometrically Determined Volume. Physical Therapy, 82, 1201-1212.

[10] Stemplewski, R., Maciaszek, J., Osinski, W. and Szeklicki, R. (2011) Test-Retest Reliability of Measurements of the 
Center of Pressure Displacement in Quiet Standing and During Maximal Voluntary Body Leaning Among Healthy Elderly Men. Journal of Human Kinetics, 28, 15-23.

[11] Corrivea, H., Hebert, R., Prince, F. and Raiche, M. (2001) Postural Control in the Elderly: An Analysis of Test-Restest and Interrater Reliability of the COPCOM Variable. Archives of Physical Medicine and Rehabilitation, 82, 80-85. http://dx.doi.org/10.1053/apmr.2001.18678

[12] Lafond, D., Corriveau, H., Hebert, R. and Prince, F. (2004) Intrasession Reliability of Center of Pressure Measures of Postural Steadiness in Healthy Elderly People. Archives of Physical Medicine and Rehabilitation, 85, 896-901. http://dx.doi.org/10.1016/j.apmr.2003.08.089

[13] Chiari, L., Rocchi, L. and Cappello, A. (2002) Stabilometric Parameters Are Affected by Anthropometry and Foot Placement. Clinical Biomechanics, 17, 666-677. http://dx.doi.org/10.1016/S0268-0033(02)00107-9

[14] Chao, J.D.M.D., Memmel, H.C.M.D., Redding, J.F.B.A., Egan, L.P.T., Odom, L.C.M.A. and Casas, L.A.M.D. (2002) Reduction Mammaplasty Is a Functional Operation, Improving Quality of Life in Symptomatic Women: A Prospective, Single-Center Breast Reduction Outcome Study. Plastic and Reconstructive Surgery, 110, 1644-1652. http://dx.doi.org/10.1097/00006534-200212000-00003

[15] Shamley, D.R., Srinanaganathan, R., Weatherall, R., Oskrochi, R., Watson, M., Ostlere, S. and Sugden, E. (2007) Changes in Shoulder Muscle Size and Activity Following Treatment for Breast Cancer. Breast Cancer Research and Treatment, 106, 19-27. http://dx.doi.org/10.1007/s10549-006-9466-7

[16] Ciesla, S. and Polom, K. (2010) The Effect of Immediate Breast Reconstruction with Becker-25 Prosthesis on the Preservation of Proper Body Posture in Patients after Mastectomy. European Journal of Surgical Oncology, 36, 625-631. http://dx.doi.org/10.1016/j.ejso.2010.05.005

[17] Perry, S.D., Mcllroy, W.E. and Maki, B.E. (2000) The Role of Plantar Cutaneous Mechanoreceptors in the Control of Compensatory Stepping Reactions Evoked by Unpredictable, Multi-Directional Perturbation. Brain Research, 877, 401-406. http://dx.doi.org/10.1016/S0006-8993(00)02712-8

[18] Winters-Stone, K.M., Torgrimson, B., Horak, F., Eisner, A., Nail, L., Leo, M.C., Chui, S. and Luoh, S.W. (2011) Identifying Factors Associated with Falls in Postmenopausal Breast Cancer Survivors: A Multi-Disciplinary Approach. Archives of Physical Medicine and Rehabilitation, 92, 646-652. http://dx.doi.org/10.1016/j.apmr.2010.10.039

[19] Wampler, M.A., Topp, K.S., Miaskowski, C., Byl, N.N., Rugo, H.S. and Hamel, K. (2007) Quantitative and Clinical description of Postural Instability in Women with Breast Cancer Treated with Taxane Chemotherapy. Archives of Physical Medicine and Rehabilitation, 88, 1002-1008. http://dx.doi.org/10.1016/j.apmr.2007.05.007

[20] Sahin, I., Iskender, S., Ozturk, S., Balaban, B. and Isik, S. (2013) Evaluation of Breast Reduction Surgery Effect on Body Posture and Gait Pattern Using Three-Dimensional Gait Analysis. Aesthetic Plastic Surgery, 37, 549-553. http://dx.doi.org/10.1007/s00266-013-0102-8

[21] Barbosa, A.F., Lavoura, P.H., Boffino, C.C., Siqueira, C.M., Costa, M.P., Lima Junior, J.E. and Tanaka, C. (2013) The Impact of Surgical Breast Reduction on the Postural Control of Women with Breast Hypertrophy. Aesthetic Plastic Surgery, 37, 321-326. http://dx.doi.org/10.1007/s00266-012-0049-1

[22] Barbosa, A.F., Raggi, G.C., Sá, C.S.C., Costa, M.P., Junior, E.L. and Tanaka, C. (2012) Postural Control in Women with Breast Hypertrophy. Clinics, 67, 757-760. http://dx.doi.org/10.6061/clinics/2012(07)09

[23] Baldini, A., Nota, A., Cravino, G., Cioffi, C., Rinaldi, A. and Cozza, P. (2013) Influence of Vision and Dental Occlusion on Body Posture in Pilots. Aviation, Space, and Environmental Medicine, 84, 823-827. http://dx.doi.org/10.3357/ASEM.3541.2013

[24] Anker, L.C., Weerdesteyn, V., van Nes, I.J., Nienhuis, B., Straatman, H. and Geurts, A.C. (2008) The Relation between Postural Stability and Weight Distribution in Healthy Subjects. Gait Posture, 27, 471-477. http://dx.doi.org/10.1016/j.gaitpost.2007.06.002

[25] Genthon, N. and Rougier, P. (2005) Influence of an Asymmetrical Body Weight Distribution on the Control of Undisturbed Upright Stance. Journal of Biomechanics, 38, 2037-2049. http://dx.doi.org/10.1016/j.jbiomech.2004.09.024 